\title{
On the Mind/Body Problem: The Theory of Essence
}

\author{
J. Kenneth Arnette, Ph.D. \\ Colorado State University
}

ABSTRACT: The classical mind/body problem can be approached empirically, using instances of the near-death experience (NDE) as experimental data. The monistic viewpoint, that the mind is the functioning of the brain, finds little support in the NDE data, while dualism, mind and body as separate entities, is consistent with NDE research to date. Comparison of the details of the NDE with predictions from theoretical cosmology shows strong similarities between the two and further strengthens the case for dualism. A theory of human nature is proposed that incorporates these similarities.

The mind/body problem has been a topic of great interest to philosophers, scientists, and psychologists throughout history. From the speculations of Plato, Aristotle, and other Greek philosophers to the dualist viewpoint of Descartes to modern psychology's material monist bent, the mind/body relationship has helped form the foundations of paradigms related to the understanding of human nature.

There are two fundamental positions one might reasonably take on the physical relationship between mind and body. I will call these the monist and dualist positions. The monist position is stated most economically as: the mind is nothing more than the biological functioning of the brain. Consider an analogy with computers. The monist might say that the brain corresponds to the central processing unit (CPU) of the computer, while the body and its organ systems are analogous to the peripherals in a computer system. The CPU serves to supervise

J. Kenneth Arnette, Ph.D. received a doctorate in Physical Chemistry from Florida State University and is currently a Ph.D. candidate in Counseling Psychology at Colorado State University. Reprint requests should be addressed to Dr. Arnette at the Department of Psychology, Colorado State University, Fort Collins, CO 80523. 
and direct the functions of the system. It takes input from the environment via the keyboard and through feedback from the peripherals; it analyzes the input from its various sources and carries out tasks in accordance with its programming, which takes the form of hardwired circuits and software commands.

In a manner similar to the CPU, the monist might propose, the brain is designed and constructed to supervise and direct the functioning of the body. The brain receives information about the environment from the senses; using both "hardwired," genetically determined structural pathways that developed through evolution, and "software" learned from the environment, the brain executes its programming based on the information it receives. In both the computer and biological cases, "mind" could be defined as the incoming information or stimuli, the accommodation and analysis of this information by the program, and the outgoing commands or responses. A generous monist might be willing to grant a certain degree of self-awareness in the biological case, stating simply that the biological program has the ability to observe its own functioning and learn from it, but that the advent of artificial intelligence in computer science has provided computers with the same ability. And the monist must insist that destruction of the brain ends the existence of the mind, just as destruction of the computer ends the program.

The dualist position states that mind and body are distinct entities that interact while the body lives and go their separate ways with the body's death. To begin, the dualist might agree to an extent with the monist's computer analogy. The brain does bear resemblance to a biological CPU, in that it represents the point at which organic "circuits" meet, interact, exchange information, and formulate action. There are genetic and environmental determinants of behavior. But, the dualist would argue, such capabilities fall far short of describing the totality of human nature, activities, and existence. The human capacity for creativity, insight, self-actualization and growth, intuition, and personal relationships goes far beyond what any computer, or animal, analogy could hope to represent. Further, the dualist might point out, there exists evidence that humans possess free will, and such a capacity could never be accurately represented by a monist perspective. To clarify the position, the dualist could appeal to another analogy: the player piano. Yes, the piano can play according to a determined pattern, without the aid of an instrumentalist; but someone capable of writing new songs can sit down at the keyboard and play tunes for which no program exists. It is the same piano in both cases; 
the question is whether anyone is sitting at the bench. The dualist says yes.

Were the monist and dualist to debate their positions, the monist would strongly object to the dualist's reasoning. The concept of an independently existing mind is inherently unscientific, the monist would say. There is no anatomical or physiological evidence for the mind's separate existence. There is no precedent in the natural world for free will or independent, non-determined thought or consciousness. Such apparently real activities are actually epiphenomenal, that is, merely by-products of physical experience. To claim that humans possess these special abilities is to distance our species from the natural order of which we are so obviously a part. The scientific principle of parsimony (Occam's Razor) demands that we trim all unnecessary speculation from our theories; the theories may be broadened only when empirical evidence leaves us no choice. Therefore, since current theories of cognition and behavior can in principle explain all human activity without resorting to the concept of independent mind, there is no justification for the dualist's point of view.

It seems, then, that the dualist's position rests merely on a hunch and a hope. There appears to be no experimental evidence capable of driving the cautious scientist to propose dualism. Occam's Razor has cut the mind out of the picture. Or has it? We should ask what kind of evidence would be sufficient to cause doubt about the monist's airtight scientific paradigm. Certainly it is difficult to conceive of an experiment that would unambiguously demonstrate the mind's existence independent of the body. Only the final experiment, the death of the body, could conceivably provide such evidence, and then only to the dead person. And none who have died have come back to convince us of the mind's continued existence, have they?

\section{The Near-Death Experience}

Raymond Moody (1975) was the first modern investigator to compile cases of the near-death experience (NDE). He found that the NDE, a subjective psychological experience, is undergone by a considerable fraction of people who are threatened with physical death but survive. The details of the NDE, discussed below, can be interpreted to support the dualist position, and Moody leaned in the dualist direction. But one must acknowledge at the outset that the NDE does not constitute the "perfect experiment," since those having an NDE do not meet perma- 
nent physical death by definition. It will become clear, however, that the NDE presents some true problems for the monist.

Moody's investigation, while a crucial first step in near-death research, lacked the scientific precision and statistical analyses that could have given his work more credibility. Kenneth Ring $(1980,1984)$, a psychologist who was inspired by Moody's book, put considerable effort into scientifically establishing the NDE as a widely experienced and largely reproducible psychological phenomenon. Ring found, among other interesting results, that the occurrence of the NDE did not depend on whether the NDEr had previously heard of the phenomenon; the NDE has occurred to those who were merely in a coma, as well as to those who were truly clinically dead, and suffered permanent brain damage as a result; and likelihood of experiencing an NDE did not depend on a person's religious faith or lack thereof.

Although each person's NDE is somewhat different, Ring was able to assemble a composite NDE from more than two hundred individual descriptions (Ring, 1984). It is important to note that individual experiences go far beyond Ring's summary; they are rich in detail and in potential for meaning. As an example, I quote from Ring's (1984, pp. $39-40$ ) report of the NDE of an anthropologist injured in an auto accident:

It seemed to be sequential in nature, more or less, I say more or less because time itself seems to have disappeared during this period. But the first thing I noticed was that I was dead ... [Could you see your body?] $\mathrm{Oh}$, yes, quite clearly. I was floating in the air above the body ... and viewing it down sort of a diagonal angle ... Then, after that, I realized that I was able to float quite easily, even though I had no intention of doing that ... Then I very quickly discovered also that not only was I floating and hence free from gravity but free also from any of the other constrictions that inhibit flight ... I could also fly at a terrific rate of speed ... and it seemed to produce a feeling of great joy and sense of actually flying in this total fashion ... Then I noticed that there was a dark area ahead of me and as I approached it, I thought that it was some sort of tunnel and immediately, without further thought, I entered into it and then flew with an even greater sensation of the joy of flight ... After what I now would imagine to be a relatively short period of time-although again time was dispensed with-I noticed a sort of circular light at a great distance which I assumed to be the end of the tunnel as I was roaring through it... And then I went through the tunnel and seemed to be in a different state. I was in different surroundings where everything seemed to be similarly illuminated by that same light and, uh, I saw other things in it, too ... a number of people... I saw my father there, who had been dead for some twenty-five years ... I also felt and 
saw of course that everyone was in a state of absolute compassion to everything else ... It seemed too that love was the major axiom that everyone automatically followed . . . Later I did feel, because of my children and the woman I was married to then, the urge to return . . . but I don't recall the trip back . . . [Did it seem like a dream?] No, it seemed nothing like a dream ... It really is a strange sensation to be in, but it does give you a feeling that you are in a kind of eternity.

This individual's account matches closely with many others provided by Moody, Ring, and several other investigators. The major details, such as viewing one's body from above, having no sense of time, being uninfluenced by gravity, entering a dark tunnel and emerging in a completely different world, are repeated time and again in NDE reports.

The nature and consistency of these details are quite intriguing. Yet despite the common, reproducible features of the NDE, this still amounts (at first thought) to subjective experience that is not objectively verifiable. There are explanations other than a mind/body dissociation that one must first address. Parsimony requires that one demonstrate the inadequacy of all biological and psychological explanatory models for the NDE before embracing a dualist interpretation.

On second thought, one might be able to devise an experiment that would lend some objectivity to the accounts of NDErs. If one could arrange for unique, purely visual stimuli to be present at the time of an NDE, and if the NDEr could, after the experience, accurately describe these stimuli without previous knowledge of them, then monist explanations would be in deep trouble. If, further, the major details of the NDE listed above were shown to be compatible with predictions made from modern physics, then the dualist position would be greatly strengthened as well.

Michael Sabom (1982), a cardiologist, read Moody's work and was skeptical of Moody's dualist interpretation of the data. Sabom began a medical investigation of the NDE at the hospital in which he worked. He compiled more than one hundred NDE cases, most of them occurring during hospitalization and many during surgery. Sabom reported many interesting cases and observations, confirming almost all aspects of Moody's and Ring's studies. But the most important portion of Sabom's work involved operating room (OR) NDEs.

While not designed as such, OR NDEs provide a version of the visual experiment mentioned above. The patient is unconscious before being brought into the OR, and is probably not familiar with equipment or specific procedures used during surgery. Thus, if a patient has an NDE 
under these conditions, the opportunity exists to compare the patient's recall of the details with the medical records and with standard medical practices. This experiment is not ideal, since a patient might be familiar enough with medicine to reconstruct the events fairly well, or may be able to hear under anesthetic and infer visual details from the sounds. Even with these flaws, the OR presents one of the best chances to evaluate the NDE objectively.

Sabom recognized this opportunity, and described in detail the experiences of ten patients who had OR NDEs and were attentive to the events in the OR during the NDE. Sabom found their memories to coincide extremely well with the medical records and procedures. For example, a man who suffered a cardiac arrest while hospitalized was able to observe in detail during his NDE the events leading to his revival. He recalled with amazing accuracy the behavior of the two meters on the defibrillator, even though he had never seen this machine in operation. Sabom verified the man's accuracy, which, Sabom said, would have required observing the defibrillator in action.

Sabom made a second major contribution to near-death research by making critical comparisons of the NDE with other psychological experiences proposed to explain the NDE in terms of monistic models. As I pointed out previously, monistic explanations must be shown to be inadequate before a dualist model can be proposed. Sabom went a long way in this direction by comparing the key features of the NDE with those of other psychological phenomena, such as autoscopic hallucinations and reactions to anesthetics. He showed that no monistic model yet proposed could account for the details of the NDE.

Given the frequency and consistency of the NDE, its uniqueness among psychological experiences, its apparent inability to be explained by monist models, and the strong medical evidence that some NDErs have observed their own revivals, one might suspect that the dualist model is the best fit to the data. At this point, however, the monist may declare that the entire question has been pushed completely beyond the realm of science. How can any aspect of the NDE be verified, understood, and accepted, the monist asks, if the NDE lies outside natural physical experience? How can we hope to integrate some dualistic interpretation of the NDE into the current body of scientific knowledge without this integration being highly suspect? The theory proposed below will, in its complete form, attempt to answer such questions in the greatest detail possible, by using as data the observations of the NDErs themselves and as a theoretical framework the latest results and ideas from chemistry and physics. 


\section{Theoretical Cosmology}

As is demonstrated each time a vehicle passes another on the highway, motion and velocity are relative quantities dependent on one's frame of reference. In ordinary life, all motion obeys this intuitive principle. It was the experimental determination early in this century that the speed of light is the same for all observers, regardless of reference frame, that stimulated Albert Einstein to consider the ramifications of such a surprising, counter-intuitive result.

The invariance of the speed of light was considered at the time of its discovery to be contradictory to the well-established principle of relativity, the concept that the general laws of physics are the same in all reference frames moving uniformly relative to each other. Einstein (1961) proposed that in fact there was no contradiction and that both these principles could be accepted as true, providing that some other cherished concepts were abandoned. He thereby derived from these two principles the special theory of relativity. The major results of this theory are (1) the time interval between two events and the distance between two points on a rigid rod depend on the state of motion of the reference body; that is, distance and time, those seemingly constant quantities, depend on the motion of the observer; (2) time can be treated mathematically as a fourth dimension equivalent to length, width, and height. Therefore, space and time lose their distinction and the resulting four-dimensional universe in which we live can be called spacetime; (3) a body with non-zero mass can never reach the speed of light, and will increase in mass as its velocity approaches the speed of light; and (4) energy and mass are equivalent to each other and can be interconverted. A great deal of experimental evidence gathered after Einstein published the special theory, not the least of which being the atomic bomb, demonstrated the theory's validity.

The special theory refers to frames of reference that are in uniform motion relative to each other. The general theory of relativity begins with the postulate that the laws of physics are the same in all (including accelerated) reference frames. This generalization paves the way for the development of the highly mathematical and highly accurate general theory. One result of the general theory is that mass can be represented as a "warp" or depression in the very fabric of spacetime. Gravity is thus a consequence of this spacetime curvature, and not a true force associated with properties of interacting bodies; when a small body is drawn to a large one, as when a meteorite falls to earth, the small body is effectively moving into a spacetime depression. A 
second result of general relativity is that, due to the curvature of spacetime by mass, light (composed of massless photons) will be influenced in its path through space by very massive objects, such as stars and galaxies. This prediction has been experimentally verified by astronomers. A third result is that the motion of clocks is influenced by gravity, so that time depends, for example, on the height of the observer above the earth. Again, this prediction is consistent with experiment.

The general theory of relativity suggests that spacetime in the absence of matter is in some sense "flat," at least in nearby (local) regions of the universe, and deviates from "flatness" under the influence of mass. This deviation increases as the mass of an object increases. The existence of this or any other type of curvature of spacetime implies the existence of at least one dimension beyond our familiar four. In order to see this point more clearly, consider a two-dimensional analogy. A universe of two spatial dimensions would contain only length and width; it would correspond to a plane or slice through our threedimensional space. Occupants of a two-dimensional universe would have absolutely no concept of the third spatial dimension, height. From the occupants' point of view, a body entering their world from the third dimension would appear literally from nowhere, and simply vanish when it left. It would be difficult to convince these occupants of the third dimension's existence, since it could not be seen or detected in any way. Similarly, the concept of curvature of the two-dimensional universe would be quite foreign to the occupants, and thus very difficult to conceptualize.

Now suppose that this two-dimensional universe is in fact curved on a very large (non-local) scale. This curvature could be represented by placing a sheet of paper on a table, lifting one end of the sheet, and curling the paper so that one end is on the table and the other end lies above the first. The occupants of this universe are constrained to move and perceive only in the two available dimensions; they cannot look "above" their location to see "the other end" of their universe, since "above" has no reality to them. To those living in three-dimensional space, this curvature of the two-dimensional universe is real and observable, but only because of the existence of the third dimension.

General relativity proposes that our own spacetime is curved on a very large scale, as in the analogy above, as well as possessing local curvature under the influence of mass. These curvatures are imperceptible to us, but their existence can be inferred from the theory. Therefore, the existence of higher dimensions can also be inferred. But we 
need not stop at these inferences, for relativity makes further predictions. One prediction concerns the aging and eventual demise of stars.

At the end of a star's life, when its hydrogen fuel is nearly spent, the star undergoes radical changes. These changes can range from a dramatic cooling and loss of luminosity to death by supernova, given sufficient initial star mass. If the star is large enough, the core remaining after the supernova will eventually collapse under its own gravity, according to general relativity, into an extremely small and infinitely dense collection of matter called a black hole. The spacetime representation of a black hole is an infinitely deep depression (a "hole") in the spacetime continuum, causing such severe curvature that even the massless photons of light that come within a certain distance cannot escape, thus giving the hole its black appearance. This phenomenon is predicted by the equations of relativity, and evidence of black holes has been found at the center of several galaxies. The case for higher dimensions is therefore strengthened.

The same equations that predict and describe black holes also predict another type of spacetime distortion: the wormhole, an entity of intense interest to theoretical cosmologists in the last decade. The wormhole can exist in association with a black hole, or independently. It can constitute a pathway, a shortcut as it were, between distant parts of our universe. Within the analogy of our two-dimensional universe described above, passage through a wormhole could conceivably allow one to move from one end of the sheet of paper to the other without passing any of the points in-between (in the two-dimensional universe). Again, such a thing is possible only because a higher dimension is available. A representation similar to our two-dimensional model with a connecting wormhole is given in Figure 1.

The equations of general relativity allow, in addition to wormholes connecting different parts of our universe, wormholes that connect ours with one or more other universes. The analogy here is to two planar universes connected by a wormhole (see Figure 1). This type of wormhole explicitly acknowledges the higher dimensions implied in the earlier results of relativity, and invites one to imagine leaving our spacetime for other destinations as yet unnamed. Note that the wormhole shown in Figure 1 displays cylindrical symmetry and funnel-like openings at both ends.

The greatest cosmologist since Einstein is Stephen Hawking. In his description of cosmology, Hawking (1988, p. 147) proposed that: "Our subjective sense of the direction of time, the psychological arrow of time, is therefore determined within our brain by the thermodynamic 


\section{Figure 1}

A three-dimensional wormhole connecting two planar spacetimes, one "above" and one "below" the wormhole.

One mentally allows the "mouths" of the wormhole to smoothly approach and coalesce with parallel two-dimensional surfaces, which can represent either two separate universes or two distant regions of a single universe. Adapted from Misner, Thorne, and Wheeler (1973).

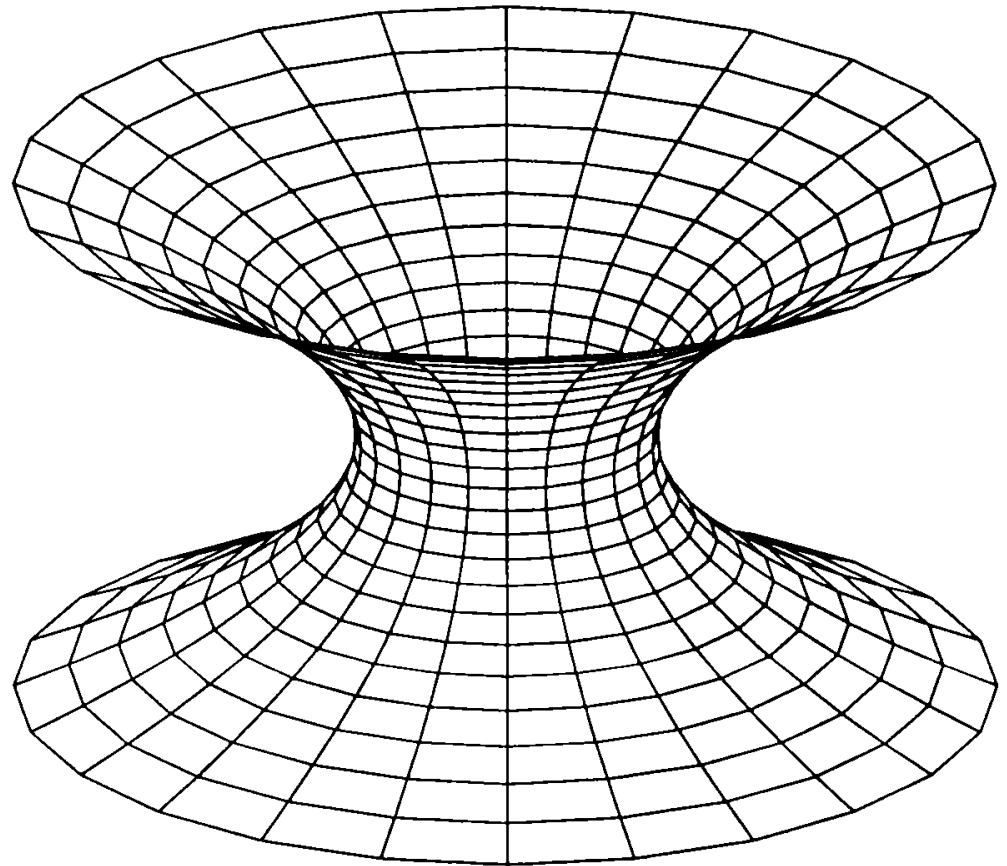

arrow of time... we must remember things in the order in which entropy increases." The "thermodynamic arrow of time" is determined by the universe's direction of motion (expansion). Our sense of time, then, is a result of our being part of the material universe. Thus, if one were to leave this universe for another through a wormhole, it seems one would leave behind this universe's definition of time, space, and gravity as well.

To briefly summarize, the field of modern theoretical cosmology began with the musings of Einstein over two apparently true but apparently contradictory principles in experimental physics. His resolution of the 
seeming conflict challenged normal modes of thought and the commonsense assumptions of the time. The resulting theories of special and general relativity provided empirically testable predictions, many of which have been shown to be correct. In the process of trying to understand our universe and its physical laws, Einstein and those who continued his work were led to a paradigm that suggests the existence of other universes. Thus, it appears that the nature of our existence and our world may be intimately intertwined with worlds beyond.

The physicists working on such problems readily admit that there is as yet no evidence for the existence of wormholes. That fact has not, however, slowed research on the size, number, distribution, destination, and traversability of wormholes. Thus there are predictions from physics on these various questions, but with no apparent means of empirically testing the predictions. The theory of essence offers the possibility of such testing by drawing together the fields of cosmology and near-death research.

\section{The Theory of Essence}

It is instructive to read carefully the account of the anthropologist's NDE quoted earlier, while referring to Figure 1 . Commencing with the death of his body, the NDEr's mind or consciousness seemed to dissociate from the body. He was able to view the body from an exterior location, and found himself to be free from both time and gravity. He realized that his motion was unrestrained by physical laws and was limited only by his thoughts. During his "flight," the NDEr came upon "a dark area" that was the entrance to a tunnel-like passageway. At the end, after traversing the tunnel, he observed a circular opening into a completely different reality.

The correspondence of the NDEr's description with the representation in Figure 1 is very strong. An observer free to leave this universe and travel interdimensionally would leave gravity and time behind as well. Coming upon a wormhole, the observer would see a hole in spacetime that one could reasonably suppose to be dark. The use of the word "tunnel" to describe the wormhole is valid, since both tunnels and wormholes have cylindrical symmetry. As predicted by relativity, the ends of the wormhole would appear "circular" from the inside. Emerging on the other side of the wormhole, the observer would be exposed to a totally new and (perhaps initially) unfamiliar universe where his or her previous conceptions of time, and indeed of life, would not necessarily apply. 
In order to emphasize the similarities between the NDE tunnel and the mathematically predicted wormhole, I quote the tunnel experiences of a few NDErs below. It is important to remember that each of these observations was made independently and that the experience is very hard to put into words. Given these facts, the agreement among the descriptions is impressive (the emphases are those of the speakers themselves):

Well, it was like night. It was dark. It was dark. But it was like, like [pause] like in the dark sky. Space. Dark. And it was-there weren't any things around. No stars or objects around. (Ring, 1980, p. 55)

I do remember thinking to myself that I was dying. And I felt I was floating through a tunnel... When I say tunnel, the only thing I can think of is-you know, those sewer pipes, those big pipes they put in? It was round like that, but it was enormous. I really couldn't see the edges of it; I got the feeling that it was round. (Ring, 1980, p. 54)

The first thing I remember was a tremendous rushing sound, a tremendous [searching for words]... It's very hard to find the right words to describe. The closest thing that I could possibly associate it with is, possibly, the sound of a tornado-a tremendous, gushing wind, but almost pulling me. And I was being pulled into a narrow point from a wide area. (Sort of going into a funnel?) Yes! Yes. And it was [pause] nothing painful. There was nothing frightening about it. It was just something that I felt I gave myself into completely. And it felt good. (Ring, 1980, p. 63)

I felt as though I was-well, that's the hard part to explain-like you're floating. Like you're there and, believe it or not, the color isthere is no color [pause] it's like a darkness. (Did the darkness have a shape of any kind?) It was empty. Yeah, that's it. Space. Just nothing. Nothing but something. It's like trying to describe the end of the universe. (Ring, 1980, p. 55)

The sense of space without matter, the complete darkness, the funnellike entrance, and the cylindrical shape are all consistent with the idea of interdimensional travel as represented by relativity theory. Indeed, as the last quoted NDEr said, it is like trying to describe the end of the universe, for that is exactly what it is.

At this juncture, I find it very easy and natural to seriously consider the dualist position on the mind/body problem. Such consideration is necessary if potential answers to the difficult questions concerning the NDE are to be entertained. The NDE evidence itself is strongly suggestive of dualism, and when combined with the results of relativity the argument becomes compelling. There are very obvious parallels with many forms of religion; dissociated "mind" or "consciousness" could 
easily be called "soul" or "spirit." To avoid the religious and psychological connotations of all these terms, I will refer to the dissociated human consciousness as the "essence," since it obviously contains the cognitions, memories, emotions, and other essential components of the human personality.

The theory of essence, then, states that human nature is dualistic. While in this universe, we are composed both of a physical body and an essence, which is the seat of consciousness and thought. Upon the death of the body, the essence is disengaged from this spacetime and travels via a connecting wormhole to locales imperceptible to us while in physical form. Furthermore, rather than defying scientific understanding, the essence has some attributes and makes observations that are consistent with known principles of science and with predictions of modern physics. A full realization of the theory, which is currently under development, will place these attributes and observations in a rational, unified scientific context.

\section{Conclusion}

The maturation of both near-death research and cosmological theory offers science its first opportunity to ask and possibly answer questions that penetrate to the very core of human nature. The theory I have outlined goes beyond speculation and dogma and attempts a synthesis of two apparently disparate disciplines while being true to the scientific method.

The theory of essence in its fully realized form will provide predictions that can be tested from two directions. First, NDE data may be used to rule out or support the results of cosmological theories that have so far proved impossible to test experimentally. It is conceivable that physicists may be able to refine their models as a result. Second, physicists possibly can derive predictions based specifically on the concept of essence; these predictions can then be compared with the NDE data. Such developments lie far into the future, but are very exciting to contemplate.

\section{References}

Einstein, A. (1961). Relativity. New York, NY: Crown.

Hawking, S. (1988). A brief history of time: From the big bang to black holes. New York, NY: Bantam.

Misner, C., Thorne, K., and Wheeler, J. (1973). Gravitation. San Francisco, CA: Freeman and Company.

Moody, R. A., Jr. (1975), Life after life Covington, GA: Mockingbird. 
Ring, K. (1980). Life at death: A scientific investigation of the near-death experience. New York, NY: Coward, McCann and Geoghegan.

Ring, K. (1984). Heading toward omega: In search of the meaning of the near-death experience. New York, NY: William Morrow.

Sabom, M. B. (1982). Recollections of death: A medical investigation New York, NY: Harper and Row. 